\title{
Matching Readers' Preferences and Reading Skills with Appropriate Web Texts
}

\author{
Eleni Miltsakaki \\ University of Pennsylvania \\ Philadelphia, U.S.A. \\ elenimi@seas.upenn.edu
}

\begin{abstract}
This paper describes Read-X, a system designed to identify text that is appropriate for the reader given his thematic choices and the reading ability associated with his educational background. To our knowledge, Read-X is the first web-based system that performs real-time searches and returns results classified thematically and by reading level within seconds. To facilitate educators or students searching for reading material at specific reading levels, Read-X extracts the text from the html, pdf, doc, or $\mathrm{xml}$ format and makes available a text editor for viewing and editing the extracted text.
\end{abstract}

\section{Introduction}

The automatic analysis and categorization of web text has witnessed a booming interest due to increased text availability of different formats (txt, ppt, pdf, etc), content, genre and authorship. The web is witnessing an unprecedented explosion in text variability. Texts are contributed by users of varied reading and writing skills as opposed to the earlier days of the Internet when text was mostly published by companies or institutions. The age range of web users has also widened to include very young school and sometimes pre-school aged readers. In schools the use of the Internet is now common to many classes and homework assignments. However, while the relevance of web search results to given keywords has improved substantially over the past decade, the appropriateness of the results is uncatered for. On a keyword search for 'snakes' the same results will be given whether the user is a seven year old elementary school kid or a snake expert.

Prior work on assessing reading level includes (Heilman et al., 2007) who experiment with a system that employs grammatical features and vocabulary to predict readability. The system is part of the the REAP tutor, designed to help ESL learners improve their vocabulary skills. REAP's information retrieval system (Collins-Thompson and Callan, 2004) is based on web data that have been annotated and indexed off-line. Also, relatedly, (Schwarm and Ostendorf, 2005) use a statistical language model to train SVM classifiers to classify text for grade levels $2-5$. The classifier's precision ranges from $38 \%-75 \%$ depending on the grade level.

In this demo, we present Read-X, a system designed to evaluate if text retrieved from the web is appropriate for the intended reader. Our system analyzes web text and returns the thematic area and the expected reading difficulty of the retrieved texts. ${ }^{1}$ To our knowledge, Read-X is the first system that performs in real time a)keyword search, b)thematic classification and c)analysis of reading difficulty. Search results and analyses are returned within a few seconds to a maximum of a minute or two depending on the speed of the connection. Read- $X$ is enhanced with an added component which predicts difficult vocabulary given the user's educational level and familiarity with specific thematic areas.

\section{Web search and text classification}

Internet search. Read-X uses Yahoo! Web Services to execute the keyword search. When the search button is clicked or the enter key depressed after typing in a keyword, Read-X sends a search request to Yahoo! including the keywords and, optionally, the number of results to return.

Text extraction. The html, xml, doc or pdf documents stored at each URL are then extracted in a cleaned-up, tag-free, text format. At this stage a decision is made as to whether a web page contains reading material and not "junk". This is a non-trivial task. (Petersen and Ostendorf, 2006) use a classifier for this task with moderate success. We "read" the structure of the html text to decide if the content is appropriate and when in doubt, we

\footnotetext{
${ }^{1} \mathrm{~A}$ demo video can be accessed at the blogsite www.eacl08demo.blogspot.com.
} 


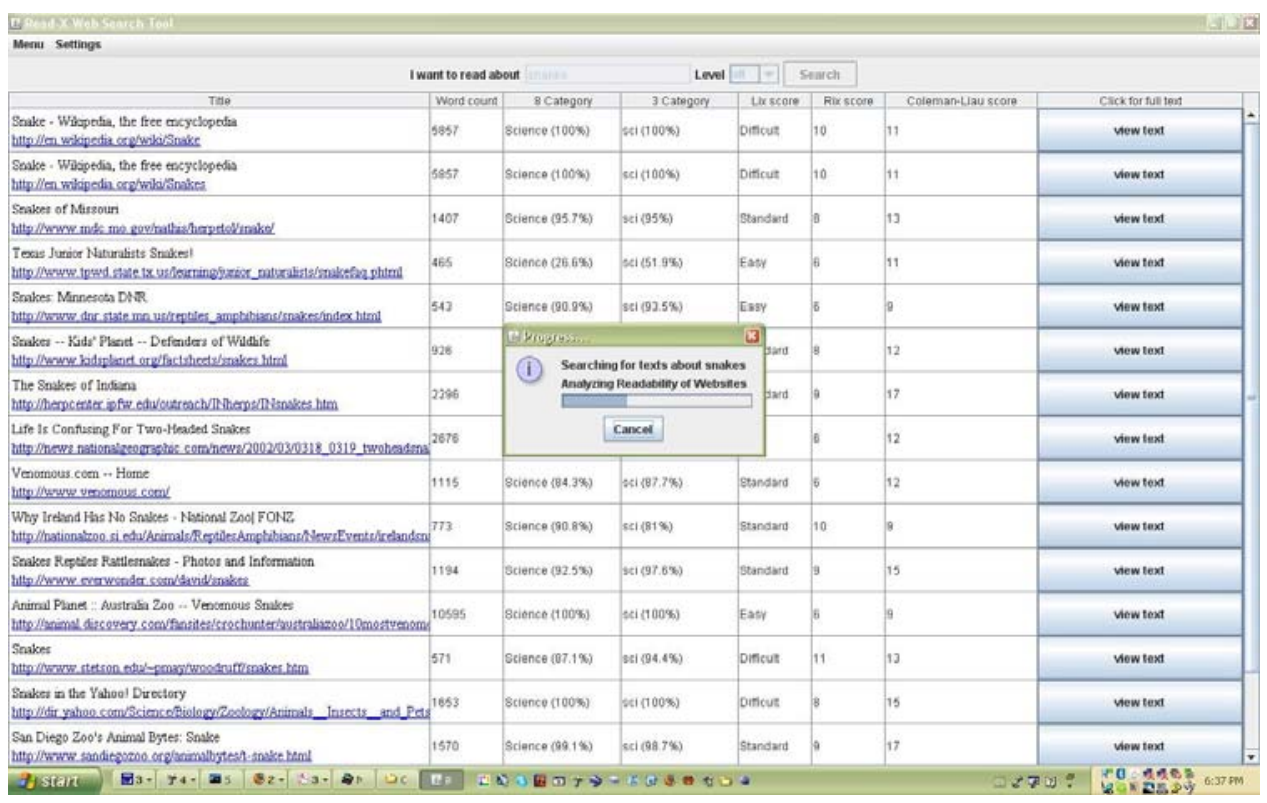

Figure 1: Search results and analysis of readability

err on the side of throwing out potentially useful content.

Readability analysis. For printed materials, there are a number of readability formulas used to measure the difficulty of a given text; the New Dale-Chall Readability Formula, The Fry Readability Formula, the Gunning-Fog Index, the Automated Readability Index, and the Flesch Kincaid Reading Ease Formula are a few examples (see (DuBay, 2007) for an overview and references). Usually, these formulas count the number of syllables, long sentences, or difficult words in randomly selected passages of the text. To automate the process of readability analysis, we chose three Readability algorithms: Lix, RIX, see (Anderson, 1983), and Coleman-Liau, (Coleman and Liau, 1975), which were best suited for fast calculation and provide the user with either an approximate grade level for the text or a readability classification of very easy, easy, standard, difficult or very difficult. When each text is analyzed, the following statistics are computed: total number of sentences, total number of words, total number of long words (seven or more characters), and total number of letters in the text. Steps have been taken to develop more sophisticated measures for future implementations. Our current research aims at implementing more sophisticated reading difficulty measures, including reader's familiarity with the topic, metrics of propositional density and discourse coherence, without compromising speed of

\begin{tabular}{l|l|l|l|}
\hline Formula & r3 & r4 & r5 \\
\hline Lix & $10.2(9-11)$ & $11.7(10-13)$ & $11.1(9-12)$ \\
\hline RIX & $10.2(8-13)$ & $12.3(10-13)$ & $11.5(10-13)$ \\
\hline Coleman-Liau & $11.65(9.2-13.3)$ & $12.67(12.2-13.1)$ & $12.6(11.4-14.1)$ \\
\hline All & 10.6 & 12.3 & 11.7 \\
\hline
\end{tabular}

Table 1: Comparison of scores from three readability formulae.

processing.

To evaluate the performance of the reading scores we used as groundtruth a corpus of webtexts classified for readability levels $r 3, r 4, r 5$ corresponding to grade levels 7-8, 9-10, and 11-13 respectively. ${ }^{2}$ The content of the corpus is a collection of web-sites with educational content, picked by secondary education teachers. For 90 documents, randomly selected from levels 3-5 (30 per level), we computed the scores predicted by Lix, RIX and Coleman-Liau.

The average scores assigned by the three formulas are shown in Table (1). The numbers in parentheses show the range of scores assigned by each formula for the collection of documents under each reading level. The average score of all formulas for $\mathrm{r} 3$ is 10.6 which is sufficiently differentiated from the average 12.3. for $\mathrm{r} 4$. The average score of all formulas for $r 5$, however, is 11.7 , which cannot be used to differentiate $\mathrm{r} 4$ from $\mathrm{r} 5$. These results indicate that at least by comparison to the data in

\footnotetext{
${ }^{2}$ With the exception of Spache and Powers-Sumner-Kearl test, all other readability formulas are not designed for low grade readability levels.
} 


\begin{tabular}{l|l|l|}
\hline Classifier & Basic categories & Subcategories \\
\hline Naive Bayes & $66 \%$ & $30 \%$ \\
\hline MaxEnt & $78 \%$ & $66 \%$ \\
\hline MIRA & $76 \%$ & $58 \%$ \\
\hline
\end{tabular}

Table 2: Performance of text classifiers.

our corpus, the formulas can make reasonable distinctions between middle school and high school grades but they cannot make finer distinctions between different high-school grades. A more reliable form of evaluation is currently underway. We have designed self-paced reading experiments for different readability scores produced by five formulas (RIX, Lix, Coleman-Liau, Flesch-Kincaid and Dale-Chall). Formulas whose predictions will more closely reflect reading times for text comprehension will be preferred and form the basis for a better metric in the future. In the current implementation, Read-X reports the scores for each formula in a separate column. Other readability features modeling aspects of discourse coherence (e.g.,(Miltsakaki and Kukich, 2004), (Barzilay and Lapata, 2008), (Bruss et al., 2004), (Pitler and Nenkova, 2008)) can also be integrated after psycholinguistic evaluation studies are completed and their computation of such features can be made in real time.

Text classification For the text classification task, we a) built a corpus of prelabeled thematic categories and b) compared the performance of three classifiers to evaluate their suitability for thematic classification task. ${ }^{3}$

We collected a corpus of approximately 3.4 million words. The corpus contains text extracted from web-pages that were previously manually classified per school subject area by educators. We organized it into a small thematic hierarchy, with three sets of labels: a) labels for supercategories, b) labels for basic categories and c) labels for subcategories. There are 3 supercategories (Literature, Science, Sports), 8 basic categories (Arts, Career and Business, Literature, Philosophy and Religion, Science, Social studies, Sports and health, Technology) and 41 subcategories (e.g., the subcategories for Literature are Art Criticism, Art History, Dance, Music, Theater).

The performance of the classifiers trained on the basic categories and subcategories data is shown

\footnotetext{
${ }^{3}$ We gratefully acknowledge MALLET, a collection of statistical NLP tools written in Java, publicly available at http://mallet.cs.umass.edu and Mark Dredze for his help installing and running MIRA on our data.
}

in Table (2). All classifiers perform reasonably well in the basic categories classification task but are outperformed by the MaxEnt classifier in both the basic categories and subcategories classifications. The supercategories classification by MaxEnt (not shown in the Table) is 93\%. As expected, the performance of the classifiers deteriorates substantially for the subcategories task. This is expected due to the large number of labels and the small size of data available for each subcategory. We expect that as we collect more data the performance of the classifiers for this task will improve.

In the demo version, Read-X uses only the MaxEnt classifier to assign thematic labels and reports results for the super categories and basic categories, which have been tested and shown to be reliable.

\section{Predicting difficult words given reader's background}

The analysis of reading difficulty based on standard readability formulas gives a quick and easy way to measure reading difficulty but these formulas lack sophistication and sensitivity to the abilities and background of readers. They are reasonably good at making rough distinctions between -standardly defined- middle, high-school or college levels but they fall short in predicting reading ease or difficulty for specific readers. For example, a reader who is familiar with literary texts will have less difficulty reading new literary text than a reader, with a similar educational background, who has never read any literary works. In this section, we discuss the first step we have taken towards making more reliable evaluations of text readability given the profile of the reader.

Readers who are familiar with specific thematic areas, are more likely to know vocabulary that is recurring in these areas. So, if we have vocabulary frequency counts per thematic area, we are in a better position to predict difficult words for specific readers given their reading profiles. Vocabulary frequency lists are often used by test developers as an indicator of text difficulty, based on the assumption that less frequent words are more likely to be unknown. However, these lists are built from a variety of themes and cannot be customized for the reader. We have computed vocabulary frequencies for all the basic thematic categories in our corpus. The top 10 most frequent words per supercategory are shown in Table (3). 


\begin{tabular}{|l|l|l|l|l|l|l|l|}
\hline \hline Arts & Career and Business & Literature & Philosophy & Science & Social Studies & Sports, Health & Technology \\
\hline \hline Word Freq & Word Freq & Word Freq & Word Freq t & Word Freq & Word Freq & Word Freq & Word Freq \\
\hline musical 166 & product 257 & seemed 1398 & argument 174 & trees 831 & behavior 258 & players 508 & software 584 \\
\hline leonardo 166 & income 205 & myself 1257 & knowledge 158 & bacteria 641 & states 247 & league 443 & computer 432 \\
\hline instrument 155 & market 194 & friend 1255 & augustine 148 & used 560 & psychoanalytic 222 & player 435 & site 333 \\
\hline horn 149 & price 182 & looked 1231 & belief 141 & growth 486 & social 198 & soccer 396 & video 308 \\
\hline banjo 128 & cash 178 & things 1153 & memory 130 & acid 476 & clemency 167 & football 359 & games 303 \\
\hline american 122 & analysis 171 & caesar 1059 & truth 130 & years 472 & psychology 157 & games 320 & used 220 \\
\hline used 119 & resources 165 & going 1051 & logic 129 & alfalfa 386 & psychotherapy 147 & teams 292 & systems 200 \\
\hline nature 111 & positioning 164 & having 1050 & things 125 & crop 368 & united 132 & national 273 & programming 174 \\
\hline artist 104 & used 153 & asked 1023 & existence 115 & species 341 & society 131 & years 263 & using 172 \\
\hline wright 98 & sales 151 & indeed 995 & informal 113 & acre 332 & court 113 & season 224 & engineering 170 \\
\hline \hline
\end{tabular}

Table 3: 10 top most frequent words per thematic category.

Vocabulary frequencies per grade level have also been computed but they are not shown here.

We have added a special component to the Read-X architecture, which is designed to predict unknown vocabulary given the reader's educational background or familiarity with one (or more) of the basic themes. The interface allows you to select a web search result for further analysis. The user can customize vocabulary difficulty predictions by selecting the desired grade or theme. Then, the text is analyzed and, in a few seconds, it returns the results of the analysis. The vocabulary evaluator checks the vocabulary frequency of the words in the text and highlights the words that do not rank high in the vocabulary frequency index for the chosen categories (grade or theme). The highlighted words are clickable. When they are clicked, the entry information from WordNet appears on the right panel. The system has not been evaluated yet so some tuning will be required to determine the optimal cut-off frequency point for highlighting words.

\section{Future work}

A major obstacle in developing better readability models is the lack of reliable 'groundtruth' data. Annotated data are very scarce but even such data are only partially useful as it is not known if interannotator agreement for readability levels would be high. To address this issue we are currently running a battery of self-paced reading and eyetracking experiments a) to evaluate which, if any, readability formulas accurately predict differences in reading times b)to test new hypotheses about possible factors affecting the perceived difficulty of a text, including vocabulary familiarity, propositional density and discourse coherence.

\section{Acknowledgments}

Audrey Troutt developed the software for Read$\mathrm{X}$ under a GAPSA Provost's Award for Interdisci- plinary Innovation, University of Pennsylvania.

\section{References}

Jonathan Anderson. 1983. Lix and rix: Variations of a littleknown readability index. Journal of Reading, 26(6):490496.

Regina Barzilay and Mirella Lapata. 2008. Modeling local coherence: An entity-based approach. Computational Linguistics.

M. Bruss, M. J. Albers, and D. S. McNamara. 2004. Changes in scientific articles over two hundred years: A coh-metrix analysis. In Proceedings of the 22nd Annual International Conference on Design of Communication: the Engineering of Quality Documentation, pages 104-109. New York: ACM Press.

M Coleman and T. Liau. 1975. A computer readability formula designed for machine scoring. Journal of Applied Psychology, 60:283-284.

K. Collins-Thompson and J. Callan. 2004. Information retrieval for language tutoring: An overview of the REAP project. In Proceedings of the Twenty Seventh Annual International ACM SIGIR Conference on Research and Development in Information Retrieval (poster descritpion.

William DuBay. 2007. Smart Language: Readers, Readability, and the Grading of Text. BookSurge Publishing. overview of readability formulas and references.

M. Heilman, K. Collins-Thompson, J. Callan, and M. Eskenazi. 2007. Combining lexical and grammatical features to improve readability measures for first and second language texts. In Proceedings of the Human Language Technology Conference. Rochester, NY.

Eleni Miltsakaki and Karen Kukich. 2004. Evaluation of text coherence for electronic essay scoring systems. Natural Language Engineering, 10(1).

Sarah Petersen and Mari Ostendorf. 2006. Assessing the reading level of web pages. In Proceedings of Interspeech 2006 (poster), pages 833-836.

Emily Pitler and Ani Nenkova. 2008. Revisiting readability: A unified framework for predicting text quality. In Proceedings of EMNLP, 2008.

Sarah E. Schwarm and Mari Ostendorf. 2005. Reading level assessment using support vector machines and statistical language models. In $A C L$ '05: Proceedings of the 43rd Annual Meeting on Association for Computational Linguistics, pages 523-530. 